\title{
BMJ Open Precarious employment, business performance and occupational injuries: a study protocol of a register-based Swedish project
}

\author{
Cecilia Orellana, ${ }^{1}$ Bertina Kreshpaj, ${ }^{1}$ Gun Johansson, ${ }^{1,2}$ Bo Burström, ${ }^{3}$ \\ Katarina Kjellberg, ${ }^{1,2}$ Tomas Hemmingsson, ${ }^{1,4}$ Magnus Axén, ${ }^{5}$ Letitia Davis, ${ }^{6}$ \\ David Wegman, ${ }^{7}$ Theo Bodin ${ }^{1,2}$
}

To cite: Orellana $\mathrm{C}$,

Kreshpaj B, Johansson G, et al. Precarious employment, business performance and occupational injuries: a study protocol of a register-based Swedish project. BMJ Open 2019;9:e026091. doi:10.1136/ bmjopen-2018-026091

- Prepublication history for this paper is available online. To view these files please visit the journal online (http://dx.doi. org/10.1136/bmjopen-2018026091).

Received 16 August 2018 Revised 17 December 2018 Accepted 18 December 2018

Check for updates

(C) Author(s) (or their employer(s)) 2019. Re-use permitted under CC BY-NC. No commercial re-use. See rights and permissions. Published by BMJ.

For numbered affiliations see end of article.

Correspondence to Dr Cecilia Orellana; cecilia.orellana@ki.se

\section{ABSTRACT}

Introduction There is uncertainty regarding the trends in occupational injuries (Ols) in Sweden due to a significant and increasing problem with under-reporting to injury registers. Under-reporting, in general, is likely to be exacerbated by the rise in precarious employment (PE), a set of unfavourable employment characteristics that would benefit from formal definition and study. PE and global trends are believed also to affect companies and their commitment to health and safety. The present study attempts to bridge these knowledge gaps and presents a study protocol for planned studies, with three main objectives: first, to review the literature for definitions of PE emphasising those that are multidimensional and operationalise components in routinely collected register data; second, to estimate the under-reporting of $0 \mathrm{l}$ in Swedish registers and third, using results from the first objective, to conduct large, register-based prospective studies, designed to measure effect sizes and interactions between $\mathrm{PE}$, business performance and $\mathrm{OI}$.

Methods and analysis First, a scientific literature review will be conducted, including scientific databases and grey literature. Second, data from two major Ol registers will be used to estimate the magnitude of under-reporting using capture-recapture methodology. Finally, all residents aged 18-65 in Sweden with any registered income during 20032015 will be included. Data sources encompass Swedish population and labour market registers with linkage to both the main 0 l register with national coverage and hospital records. Trends in $\mathrm{PE}$ and $\mathrm{OI}$ will be explored, together with risk of OI associated with PE and business performance. Ethics and dissemination The project has been approved by the Regional Ethics Committee, Stockholm (dnr: 2016/2325-31; 2017/2173-32). Dissemination of study results will include a series of peer-reviewed papers, at least one PhD thesis and one report in Swedish, engaging relevant stakeholders. Results will be presented in national and international conferences and through press releases to mass media.

\section{INTRODUCTION}

Setting priorities for workplace health and safety research depends on accurate and reliable accident and injury data. In Norway
Strengths and limitations of this study

- We present a systematic approach to operationalisation of the arising social determinant of health; precarious employment. This effort is much needed and is likely to be valuable to the research community.

- The use of high-quality, nationwide register data covering the total working population provides power to the study and virtually zero loss to follow-up.

- A wide range of variables from different sources, together with the long follow-up period will enable us to adjust for confounders and apply longitudinal designs, mitigating several sources of bias.

- There exists a potential risk of misclassification for both exposure and outcome, due to factors such as data availability and self-reporting.

- For the under-reporting of occupational injuries, the main limitation refers to differences in the coverage of the different data sources, which may limit our ability to make good estimates in some labour market sectors.

and Denmark, studies suggest that as few as $9 \%-26 \%$ of all occupational accidents reaching hospitals are also reported to the national occupational injury (OI) registers. ${ }^{1}$ There is uncertainty regarding the trends in OIs in Sweden due to under-reporting to Swedish injury registers. ${ }^{3} 4$ It is known that larger workplaces in Sweden generally have better routines for reporting OIs than smaller companies. ${ }^{4}$ Among the latter, there is a widespread poor awareness that reporting of OIs is mandatory. No systematic analysis of the magnitude of under-reporting and factors associated with this problem has been performed.

The problem with under-reporting could be exacerbated by the rise in precarious employment (PE), non-standard employment relations encompassing short-term and temporary contracts, as well as powerlessness, 
vulnerability, employment insecurity and insufficient wages. There is no internationally accepted definition of $\mathrm{PE}$, but several multidimensional constructs have been proposed. $^{5-7}$

There is a reason to believe that precariously employed workers are less likely to report OIs due to lack of knowledge, education, unionisation and empowerment in exercising rights. Precariously employed workers are also likely to be at higher risk of OI. A recent review by our group 8 supports an association between some of the dimensions of PE and OI, most notably for multiple jobholders ${ }^{9-12}$ and employees of temporary agencies or subcontractors at the same worksite. ${ }^{13-15}$ Results for employees on fixedterm contracts were inconclusive.

The rise in non-standard employment relations is a trend in many countries in all stages of development and Sweden is no exception. Here, temporary employment rose during the 1990s crisis and is especially common among young adults, where the proportion on temporary contracts is $65 \%$ among both men and women aged 20-34. ${ }^{16} \mathrm{PE}$ and business performance are also likely to be intertwined. International trends in management and increased competition affect companies and their commitment to both health and safety and good employment conditions. In order to stay economically competitive and to earn maximum profits, it has been found that construction contractors only execute basic safety measures and eliminate many important hazard prevention training programmes during project implementation. ${ }^{17}$ Others have found that when the financial condition of a firm deteriorates, then it is likely to adopt policies that will lead to an increase in safety violations, accident rate and environmental hazards. ${ }^{18}$ In contrast, companies that prosper could be more likely to invest in occupational health and safety. In a study of US coal mining companies, a $10 \%$ increase in real total revenue per hour worked was associated with $0.9 \%$ decrease in the incidence rates of all reported injuries. ${ }^{19}$ However, the relationship between business performance and the risk of OI remains largely unstudied, highlighting the importance of such a study.

Research regarding the association between PE, business performance and OIs faces several challenges. The employer-employee relationship is increasingly complex and there is mounting evidence that a single variable such as temporary employment or job insecurity is not enough to explore this relationship and its association to health outcomes. ${ }^{8}{ }^{20} 21$ A multidimensional approach is needed, but the variety in definitions used makes comparison between studies and countries difficult. Which key business indicators that are useful in researching precarious businesses in relation to OIs are unknown.

To address these challenges, the first objective of this project is to review the literature for multidimensional definitions of PE and similar constructs and identify its main components. We will then suggest ways to operationalise these components in routinely collected register data.
There is a pressing need for better injury statistics in order to set priorities for prevention and future research. Therefore, the second objective of this study is to estimate the magnitude of under-reporting of injuries in Swedish registers and investigate which factors are related to this.

Finally, the third objective of this project is to use the results from our methodological work in the first objective to conduct large, register-based prospective studies, designed to identify trends on the labour market and measure effect sizes and interactions of the relationship between PE, key business indicators and OIs as well as their interaction with sociodemographic and economic indicators.

In this protocol, we provide an overview of the data sources and methods to be used in the project.

\section{Specific research questions}

Studies are planned based on specific research questions (RQs) aligned to the overarching objectives above.

\section{Objective 1}

RQ1. How has PE been previously defined and how can this be operationalised in Swedish registers?

Objective 2

RQ2. What is the magnitude of under-reporting of OI in Sweden 2013, and is under-reporting differential with respect to:

A. Individual factors such as age, sex, educational level and PE status.

B. Organisational-level factors such as company size, industry or sector, gender composition, age composition and proportion of immigrant workers.

C. Injury severity and cause of accident.

Objective 3

RQ3. What are the trends in PE in the Swedish labour market?

RQ4. What are the OI trends over time for precarious workers and organisations or industries that go through economic change?

RQ5. Are precarious workers at higher risk of OI compared with others?

RQ6. Are changes in key business indicators risk factors for occupational accidents over short-term and long-term periods?

\section{METHODS AND ANALYSIS}

\section{Patient and public involvement}

Patients and/or members of the public have not been involved in the design of this study. Results from scientific publications will be shared with stakeholders, policy-makers and social partners.

\section{Description of data sources}

This study includes all residents aged 18-65 in Sweden with any registered income for at least 1 year, from 1 January 2003 to 31 December 2015. For the main analysis, this 
cumulatively amounts to approximately 7000000 individuals over the years 2003-2015. This project will use the Longitudinal Integration Database for Health Insurance and Labour Market Studies (LISA) with linkage to the Information System on Occupational Injuries (ISA) and hospital records, described in greater detail in tables 1 and 2. We will use the unique personal identity number assigned to each resident in Sweden to link information from all the registers used in this project.

Statistics Sweden has removed the original personal identity number and replaced with a new, unique and unidentifiable identification number, thus ensuring the confidentiality of the information.

Apart from the data sources described above, for the second objective, we will also include OIs that occurred in the year 2013 using data from AFA Insurance, an organisation owned by the Swedish labour market parties (table 1).

\section{Main variables}

\section{PE and key business indicators}

The definition of PE will be developed through the systematic literature review, development of a functional PE definition applicable to available registers and then operationalisation in registers (RQ1). Available variables regarding $\mathrm{PE}$ and key business indicators from the OI registers are limited. Because of this, we will mainly obtain them through the LISA register (table 2).

Relevant key business indicators will be selected through discussions and workshops within the research team, which includes a business economist. There is very limited guidance in previous research on which key business indicators could be related to OIs. Under the well-founded assumption that managers make decisions (including those affecting health and safety) based on the projected future of the company, the initial work has focused on identifying key indicators of company value, as reflected by operating assets, future earnings and cash flows. Preliminary discussions suggest that return on equity, operating margin, net turnover/employee, employee costs/net turnover, solidity, operating result/ employee, employee costs/employee, financial leverage, labour costs as well as total gross and net investments are highly relevant. All these variables are available directly from LISA for all Swedish companies. Stock market prices have been contemplated but discarded since most companies are not listed. We also have ongoing work identifying which key business indicators that are useful in comparing companies across industries and within specific industries. We will most likely adopt an exploratory approach on a subset of the dataset before deciding on which indicators to use.

\section{Occupational injuries}

Information on OIs caused by an accident is being obtained from two different Swedish registers: ISA and AFA Insurance (table 1). By linking Swedish population and labour market registers together with hospital records, we will be able to characterise injuries in terms of severity and add information on potential confounders.

\section{Ol definition}

We will use the definition used in Swedish Law and which is applied by both the ISA and AFA registers. 'An OI is an injury due to accident[s], which occurred at the workplace or other place where the injured person had been for work. For an event to be counted as an accident, it is required that the course was relatively short and arose in connection with a particular event.

Injuries caused by threats, assaults, robberies are also counted as OIs. Both physical and mental injuries are counted here. Injuries such as heat stroke, frostbite, inflammation and injuries due to mechanical effects for a shorter period of time, no more than a few days, are also considered to be caused by accidents. ${ }^{22}$

Since estimating under-reporting is one of our main objectives, we will study reported OIs. In ISA, claims can only be made for a limited set of compensations (table 1). No assessment of reports is made without a claim; thus, an inclusion of claims or approved claims would be too limiting. The occurrence of false reports is thought to be very low (personal communication with the work environment authority) and is likely random. However, we will explore this issue further.

Moreover, we will exclude injuries that occurred during transit to/from work, occupational diseases and near injuries.

\section{Ol severity}

Severity of OIs will be assessed in two different ways.

Days of work lost: Data from the Swedish Social Insurance Agency provided by employers and part of the ISA and AFA registers will be used to obtain days lost from work due to sickness absence and disability pension, in connection with OIs. This information covers all employees.

Specialised care, hospitalisation and death: Specialised care, hospitalisation and death will be obtained from the National Patient Register (NPR) and cause of death register (table 2). We have limited this study to chapters $S$ and $\mathrm{T}$ in International Classification of Disease, 10th Revision (ICD-10) which contain injuries, poisoning and some other consequences of external causes. Since all diagnoses are not covered, this dataset does not include all OIs leading to specialised care, hospitalisation and death. Regarding injuries to the musculoskeletal system, it only covers fractures, dislocations and distortions. However, it will allow a subpopulation analysis of severe acute injuries in RQ2-6.

\section{Linkage of data sources}

The unique personal identity number assigned to each resident in Sweden enables the linkage of information from all the registers mentioned above. Also, there is an identification number specific for each company and workplace within companies, which will allow us to conduct a three-level analysis (individuals, workplaces and 


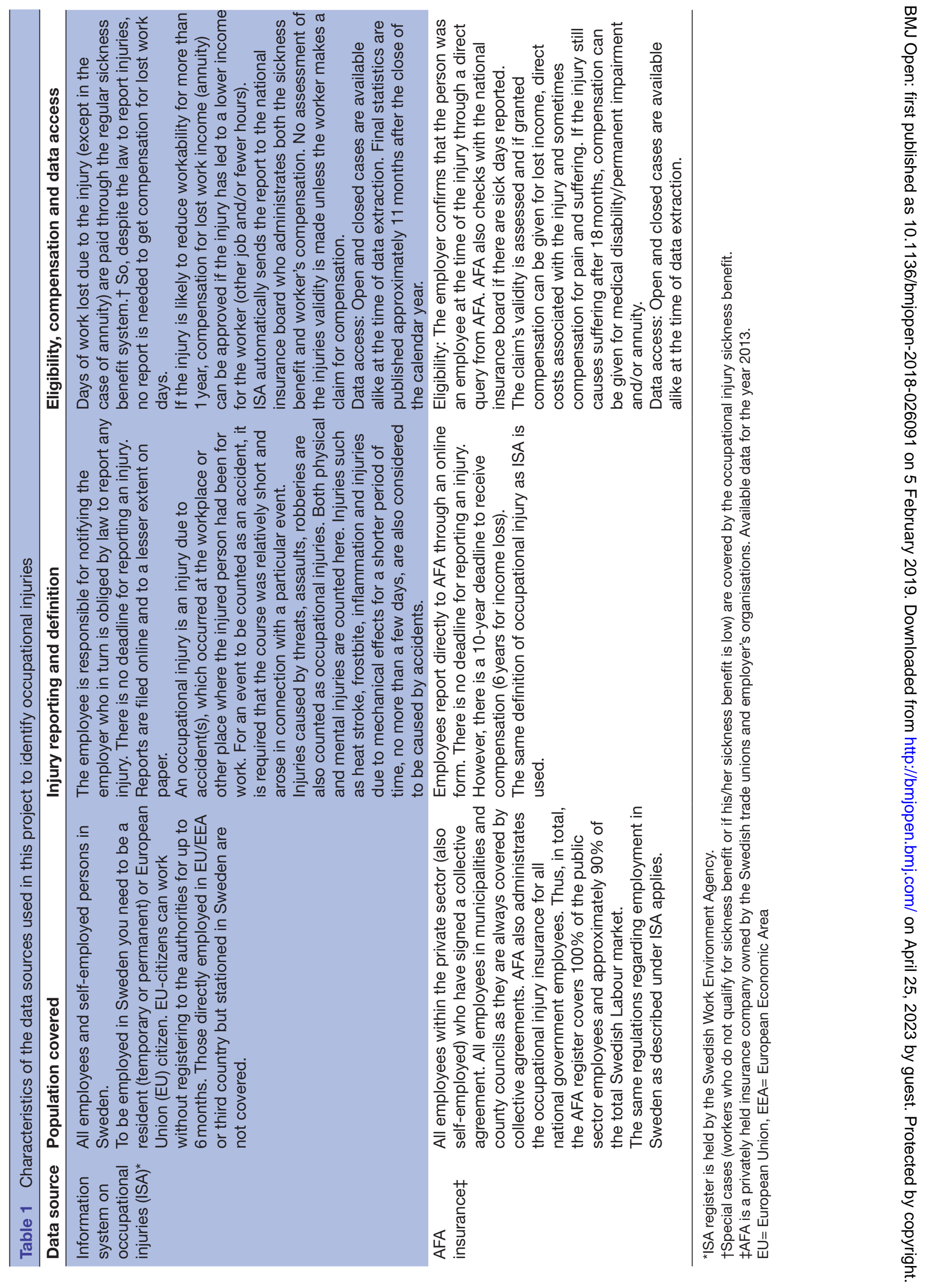


Table 2 Additional sources of data used in this project

\begin{tabular}{|c|c|c|c|}
\hline Data source & Population covered & Available variables & Timeliness \\
\hline $\begin{array}{l}\text { Longitudinal Integration } \\
\text { Database for Health } \\
\text { Insurance and Labour } \\
\text { Market Studies (LISA)* }\end{array}$ & $\begin{array}{l}\text { It holds annual registers } \\
\text { since } 1990 \text { and includes all } \\
\text { individuals } 16 \text { years of age and } \\
\text { older who were registered in } \\
\text { Sweden as of } 31 \text { December } \\
\text { for each year. }\end{array}$ & $\begin{array}{l}\text { The database integrates } \\
\text { multiple databases from the } \\
\text { labour market, educational } \\
\text { and social sectors. It contains } \\
\text { not only individual data, but } \\
\text { also connections to family, } \\
\text { companies and places of } \\
\text { employment. }\end{array}$ & $\begin{array}{l}\text { Temporal resolution: Variables are } \\
\text { on yearly basis for both individuals } \\
\text { and businesses, this applies to, for } \\
\text { example, income, revenue. } \\
\text { Multiple employers are registered } \\
\text { (total no and details on the three } \\
\text { major employers). } \\
\text { Data access: Data are compiled with } \\
\text { an 18-month lag. }\end{array}$ \\
\hline $\begin{array}{l}\text { National Patient } \\
\text { Register (NPR)† }\end{array}$ & $\begin{array}{l}\text { All visits to inpatient or } \\
\text { specialised outpatient care (ie, } \\
\text { excluding primary care). }\end{array}$ & $\begin{array}{l}\text { Our dataset includes every } \\
\text { person who was diagnosed with } \\
\text { external cause of injury (ICD-10 } \\
\text { chapters } S \text { and } T \text { ). Duration of } \\
\text { hospitalisation will be used to } \\
\text { characterise severity. }\end{array}$ & $\begin{array}{l}\text { All hospitals and specialised } \\
\text { outpatient clinics in Sweden report } \\
\text { to a central register, coverage } \\
\text { is }>95 \% \text {. } \\
\text { Data include exact date of visits } \\
\text { admission and discharge. }\end{array}$ \\
\hline
\end{tabular}

*LISA is held by Statistics Sweden.

†NPR and DR are held by the National Board of Health and Welfare.

$\ddagger$ The statistics do not include stillborns, persons who died on a temporary visit to Sweden or asylum seekers who have not yet obtained residence permits. Swedes who have emigrated and are no longer registered in Sweden are not included either.

ICD-10, International Classification of Disease, 10th Revision.

companies). This linkage will provide aggregate exposure data, such as company-specific characteristics related to individuals, and number of employees at each workplace. We will also be able to follow individuals moving between workplaces.

For objective 2 specifically, OI registers (AFA and ISA) have been linked on a \pm 7 days range basis, which means that accidents reported within a week in either register were considered to be the same. We present preliminary results for this linkage. Data from the NPR will be linked using the same criteria ( \pm 7 days range), using injury date from the OI registers and admission date from both inpatient and outpatient registers. Finally, data on sociodemographic characteristics, key business indicators and all relevant covariates will be added from the LISA register.

\section{Covariates/confounders}

We will use information from LISA register (table 2) to adjust for confounding factors, perform stratified analyses and subgroup analyses.

Individual: age, sex, educational level, income, country of birth (four groups: Sweden, other Nordic country, other European Union (EU) 25, and rest of the world) and occupation according to the Swedish Standard Classification of Occupations (1996), ${ }^{23}$ which is based on the International Standard Classification of Occupations 1988. All analyses will be stratified by sex and age.

Workplace: In addition to key business indicators, we have information on workplace level on industry
(Swedish Standard Industrial Classification),${ }^{24}$ number of employees, educational level of workforce and workplace sex distribution.

\section{Analysis plan}

Definitions and operationalisation of PE (RQ1)

We will deconstruct all definitions obtained by our literature search into single dimensions and perform a qualitative and quantitative appraisal of their appropriateness. Based on our findings, we will propose a core set of variables that should be included in a multidimensional definition of PE depending on data availability. Once these are defined, we will operationalise a multidimensional definition in Swedish registers to be able to use it in the subsequent studies and develop a job exposure matrix for PE including these variables (study 1).

\section{Under-reporting of Ol (RQ2)}

In order to estimate the magnitude of under-reporting of OI for the year 2013 (RQ6), two sources of data on OIs (ISA and AFA registers) will be used to obtain estimates by means of capture-recapture methodology (study 2). This method, based on log-linear models, has been successfully used by others and it is used to estimate the incomplete ascertainment using information from overlapping lists of cases from distinct sources. ${ }^{25}$ We will conduct stratified analyses by PE status, based on results from RQ1. 
Trends in PE and Ols (RQ3 and RQ4)

Using data from the whole study period, we will then explore trends in PE over time in Sweden, for the years 2003-2015 (as laid out in study 1). We will put special attention to changes over time for women and men, different industries and foreign born compared with Swedish born (study 3 ).

We will also explore the trends over time with regards to the risk for OIs for precarious workers and organisations or industries in economic trouble (study 4).

\section{Risk of injury associated with PE (RQ5)}

$\mathrm{PE}$, as defined through formative work in study 1 , will be the independent variable of interest in a multivariate analysis with OI as the outcome. It is well known that the risk of OI decreases with tenure at any given job. It is unlikely that there is an accumulation of risk or latency in the PE-OI relationship. We will, therefore, measure risk and outcome at the same point in time, that is, $\mathrm{PE}$ and injury in the same year. We will also explore the interaction effects of age and tenure/experience. Analysis will be adjusted for sociodemographic variables. A longitudinal dimension will be introduced by analysing the changes in risks over the time of the study period (2003-2015). Due to the large differences in risk of injury based on occupation, sector and industry, we will perform stratified analysis based on these variables. Apart from this, we will provide the population attributable fraction as part of our results.

The outcome will be stratified based on the severity of injury and differences in risk of injury severity between precarious and non-precarious employees will be explored.

\section{Risk of injury associated with key business indicators (RQ6)}

A cohort of all Swedish companies will be created. The outcome will be defined as OI per full-time employee and calculated for each year for each individual company. We will calculate short-term (1 year) and long-term (5 years) trajectories in key business indicators for each company and use these as the main independent variables of interest in a multivariate regression analysis. Stratified analysis will be carried out based on company size, industry/sector. As in RQ5, the outcome will be stratified based on the severity of injury and the population attributable fraction will be calculated.

\section{Preliminary findings}

In this protocol, we present preliminary findings based on the linkage of different data sources for the working population aged $18-65$ years, during the year 2013. The total number of OIs reported only in AFA, only in ISA as well as the overlap (presence in both registers) are shown in table 3. The linkage between AFA and ISA registers for this preliminary analysis was conducted on id-number (de-identified) and injury date, on a \pm 7 days range. There was approximately a $36 \%$ overlap between the two data sources.

\section{DISCUSSION}

In this project, we aim at operationalising PE in Swedish labour market registers and use this definition to conduct several large, register-based prospective aetiological studies, designed to measure effect sizes and interactions of the relation between $\mathrm{PE}$, business performance and OIs. Taking advantage of two separate and comprehensive reporting systems for OIs in Sweden, we will also estimate under-reporting of OIs and the factors which are related to this issue. In this protocol, we present preliminary results for the overlapping of OIs for the year 2013.

\section{Strengths and limitations}

We believe that the major strength of our project will arise from the results from the first study, that is, the operationalisation of PE. This, together with the identification of key business indicators of relevance for the association between precariousness and OIs can be considered as a valuable start point for future research investigating these factors.

By using the unique Swedish personal and organisation identification numbers from ISA and LISA, we are able to link both individuals and companies to each other and to the injury databases. The use of high-quality, nationwide register data covering the total working population provides power to the study and virtually zero loss to follow-up. A wide range of variables from different sources, together with the long follow-up period will

Table 3 Number of occupational accidents reported to either ISA, AFA or both (overlap)*, together with presence in the NPR (inpatient and outpatient), for the year 2013 in Sweden

\begin{tabular}{|c|c|c|c|c|c|c|c|c|}
\hline & \multicolumn{2}{|c|}{ All reported injuries } & \multicolumn{2}{|c|}{ Total NPR } & \multicolumn{2}{|c|}{ Inpatient } & \multicolumn{2}{|c|}{ Outpatient } \\
\hline & $\mathbf{N}$ & $\%$ & $\mathbf{N}$ & $\%$ & $\mathbf{N}$ & $\%$ & $\mathbf{N}$ & $\%$ \\
\hline ISA only & 49356 & 47.6 & 5343 & 10.8 & 368 & 0.7 & 4975 & 10.1 \\
\hline ISA and AFA & 37138 & 35.9 & 10131 & 27.3 & 1119 & 3.0 & 9012 & 24.3 \\
\hline Total & 103589 & 100.0 & 19932 & 19.2 & 1858 & 1.8 & 18074 & 17.4 \\
\hline
\end{tabular}

*Linkage of datasets conducted on id-number (de-identified) and injury date in a \pm 7 days range.

ISA, information system on occupational injuries; NPR, National Patient Register. 
enable us to adjust for confounders and apply longitudinal designs, mitigating several sources of bias. Information on sickness absence and hospitalisation allows us to characterise our outcome in terms of severity, further adding quality aspects to outcome measurement.

However, some limitations and methodological challenges should be addressed.

\section{Ol definition}

The system of self-reporting and somewhat ambiguous definition of OI introduces the risk of misclassification between OI and disease. This problem is likely to be most serious in the case of musculoskeletal disorders (MSDs) where repeated overexertion leading to an MSD could be reported as both an OI and occupational disease. For women, overexertion injuries (physical overexertion such as during heavy lifting and carrying, jerks, slips) constituted the second most common cause/type of OI with at least 1 day of sickness absence in Sweden. ${ }^{26}$ For men, it was the third most common cause. If the misclassification was random and steady over time this would pose a lesser problem, but we have reason to believe that the preference to choose between reporting MSDs as injuries or diseases might be biased by preconceptions about chances of getting a claim granted in either category and that this changes over time as a consequence of regulatory changes.

Additionally, there is a risk of including 'non-OIs' while investigating reports rather than approved claims. The rationale for not limiting the reports to approved injuries is that the rules for receiving compensation have been tightened over the last decade and we believe that on the group level, there is more consistency over time in employees' notion of what qualifies as an OI than in the assessment by the social insurance agency. In personal communication with civil servants at the Swedish Work Environment Authority, the presence of non-OI has been deemed 'an issue in the margin'.

Finally, although the occurrence of traffic-related injuries is increasing in some countries, ${ }^{27}$ we decided to exclude those from our study. Injuries that occurred during transit to/from work may be covered by car insurances and may therefore not appear in the OI registers.

\section{Injury severity}

In Sweden, sickness absence is reimbursed by the Swedish Social Insurance Agency starting on day 15. The near-universal coverage of the regular sickness insurance scheme and the additional coverage specifically for injuries makes us rather confident that we will be able to identify the most serious injuries occurring in the formal labour market. The other severity measure obtained through hospital records only covers injuries, poisoning and some other consequences of external causes ( $\mathrm{S}$ and $\mathrm{T}$ chapters in ICD-10). Thereby, we will not identify major injury categories such as acute lumbago (M45.3).

Whether we characterise severity in terms of days of hospitalisation or reimbursed days lost of work, we have a rather large difference between mild and severe cases, losing the broad spectrum of less severe injuries while severe cases can be classified in more detail.

\section{Precarious employees}

Although we have not yet operationalised PE in registers, we foresee some constraints. Among other issues, type of contract (permanent/temporary) is not registered as such and will be constructed by proxy variables obtained from LISA, such as a number of employers and number of sources of income. EU citizens stationed in Sweden and informal workers are not covered in this study, groups that are of special interest in research on PE. This is a major limitation, but the constraints in data do not allow us to study these two categories of potentially precarious workers.

Precarious employees are less likely to be covered by collective agreements, and therefore, the issue will be greatest here. We also hypothesise that they are less likely to report injuries. This will affect both our analysis of under-reporting using capture-recapture, and also the estimates for the association between PE and the risk of OIs.

\section{Key business indicators}

Previous studies have found that as the financial condition of a firm deteriorates, it is likely to adopt policies that will lead to an increase in safety violations, accident rate, and in environmental hazards ${ }^{18}$ and that when revenue increases the opposite would occur. ${ }^{19}$ The opposite might, however, be true as well. As revenue falls, the work tempo might shift downwards temporarily and the short-term effects on injuries might be positive. Lay-offs of those with least tenure may also leave a larger proportion of experienced workers which could lead to fewer injuries. Mirroring this; as revenue increases in a company, new employees come in who are at higher risk. Disentangling these effects and countereffects of changes in key business indicators will be a major challenge and will require important formative work on causal pathways and the construction of logic models prior to analysis. Due to the lack of prior research in this area, an exploratory approach using data mining or machine learning algorithms will also be applied to discover risk factors and pathways which we cannot foresee at the moment.

\section{Working hours}

When calculating the risk of OIs, a measure of working hours is needed as a denominator in order to make just comparisons. Lacking data on individual working hours in this project we have to rely on proxy variables to make estimations. Due to collective bargaining, the wage structure in Sweden is rather homogeneous, especially for blue-collar workers and white-collar workers with low skills. We are currently exploring the feasibility of using wage in combination with occupational code and industry code as well as the public registers on median salaries in certain occupations (survey-based information) to create a proxy for full-time employment based on the deviation from the median wage. 


\section{Formal/informal work}

As this study partly focuses on PE, we need to spell out that this study is only investigating the formal economy. Not including people working in the informal sector completely or partly will be one of our major limitations. According to the 2015 European Working Conditions Survey, the prevalence of informal employment for Sweden is estimated to be $5 \%$, lying below the average for the EU (10\%). ${ }^{28}$ Those who are formally employed but receive part of their salary 'under the table' will be especially at high risk of being misclassified. Also, foreign citizens working in Sweden but whose employer is registered in another country will be absent in this study. We know that these workers are very common in construction and logistics, two industries with a high risk of injury.

\section{Under-reporting}

Capture-recapture is a method that has been widely used in epidemiology to estimate the unknown size of populations. Methodological issues may appear from dependence among data sources being used to obtain estimates. ${ }^{25}$ When it comes to occupational diseases, AFA requires that a report is made first to ISA, therefore, one would expect a high level of dependency and that AFA would be completely nested in ISA. For OIs, this is not the case and there is a large proportion of injuries that are only reported to AFA. This said, the detected dependency may result in an overestimation of the true population size, and we must, therefore, treat our estimations cautiously.

Also, the AFA insurance scheme is not as comprehensive as ISAs. The ISA register covers $100 \%$ of the employees and self-employed while the AFA register covers $100 \%$ of public sector employees and all employees within the private sector who have signed a collective agreement. However, collective agreements are less common in small companies and certain industries such as hospitality where $45 \%$ of the companies have collective agreements. This will limit our ability to make good estimates in some labour market sectors.

Finally, under-reporting of OIs may be higher among precarious workers, and conversely, these workers may have a higher rate of injuries compared with non-precarious workers. To be able to observe differences in the under-reporting for precarious workers compared with non-precarious, we will conduct stratified analyses.

\section{DISSEMINATION}

The project is presently planned to result in a series of papers published in international peer-reviewed scientific journals, at least one $\mathrm{PhD}$ thesis and a report in Swedish aimed at relevant stakeholders including governmental agencies, policy-makers and social partners (employers and trade unions). Due to the richness of the data obtained and the multiple scientific approaches, we anticipate that the project will result also in further publications than those outlined in this protocol, including future collaborations. Results of public interest will be formatted as press releases and sent to Swedish and international media with support from the University press services.

\section{CONCLUSION AND POLICY IMPLICATIONS}

We believe that this project will address some of the most pressing issues related to OI surveillance and research. Despite some limitations, the inclusion of different studies within this project, using several methodologies, together with the statistical and high quality of the data will allow us to explore trends and risks in OIs in Sweden from many perspectives. The richness of our data will allow us to conduct several specialised substudies in the future which have not been outlined here, and we would be happy to receive suggestions for further studies and invitations to collaborate.

\section{Author affiliations}

${ }^{1}$ Unit of Occupational Medicine, Institute of Environmental Medicine, Karolinska Institutet, Stockholm, Sweden

${ }^{2}$ Center for Occupational and Environmental Medicine, Stockholm County Council, Stockholm, Sweden

${ }^{3}$ Department of Public Health Sciences, Equity and Health Policy Research Group, Karolinska Institutet, Stockholm, Sweden

${ }^{4}$ Department of Public Health Sciences, Stockholm University, Stockholm, Stockholm, Sweden

${ }^{5}$ Department of Business studies, Uppsala University, Uppsala, Sweden ${ }^{6}$ Occupational Health Surveillance Program, Massachusetts Department of Public Health, Boston, Massachusetts, USA

${ }^{7}$ University of Massachusetts Lowell, Lowell, Massachusetts, USA

Acknowledgements The authors acknowledge the following data providers: The Swedish Work Environment Agency, AFA insurance, Statistics Sweden and the National Board of Health and Welfare.

Contributors $\mathrm{CO}$ has drafted previous versions and the final version of the manuscript. TB is the principal investigator of the project. BK, GJ, BB, KK, TH, MA, $\mathrm{LD}$ and DW participate in the project and have contributed to the design of the project and its studies. All authors have read and approved of the final version of the manuscript.

Funding This project has been founded by grants from the Swedish Research Council for Health, Working Life and Welfare (FORTE) (Dnr: 2016-00315 and 2017-01956)

\section{Competing interests None declared.}

Patient consent for publication Not required.

Ethics approval Ethical permission for the study for the project duration was granted by the Regional Ethics Committee, Stockholm (Dnr 2016/2325-31 and 2017/2173-32)

Provenance and peer review Not commissioned; externally peer reviewed.

Open access This is an open access article distributed in accordance with the Creative Commons Attribution Non Commercial (CC BY-NC 4.0) license, which permits others to distribute, remix, adapt, build upon this work non-commercially, and license their derivative works on different terms, provided the original work is properly cited, appropriate credit is given, any changes made indicated, and the use is non-commercial. See: http://creativecommons.org/licenses/by-nc/4.0/.

\section{REFERENCES}

1. Magne Gravseth $\mathrm{H}$, Wergeland E, Lund J. Underrapportering av arbeidsskader til Arbeidstilsynet. Tidsskrift for den norske lægeforening 2003;123:2057-9.

2. Lander F. Underrapportering af arbejdsulykker Øje på arbejdsmiljøet. København: Landsorganisationen i Danmark, 2012. 
3. Swedish Work Environment Authority (Arbetsmiljöverket), Arbetsskador. Occupational accidents and work-related diseases. Stockholm: Arbetsmiljöverket, 2014.

4. Andersson L. Hälsorelaterad arbetsmiljöövervakning. Stockholm: Arbetsmiljöverket, 2014.

5. Standing G. The precariat: The new dangerous class. London: Bloomsbury Publishing, 2016

6. Vives A, Amable M, Ferrer M, et al. The Employment Precariousness Scale (EPRES): psychometric properties of a new tool for epidemiological studies among waged and salaried workers. Occup Environ Med 2010;67:548-55.

7. International Labour Organization (ILO). From precarious work to decent work: outcome document to the workers' symposium on policies and regulations to combat precarious employment. Geneva: International Labour Office, Bureau for Workers' Activities, 2012.

8. Koranyi I, Jonsson J, Rönnblad T, et al. Precarious employment and occupational accidents and injuries - a systematic review. Scand $J$ Work Environ Health 2018;44:341-50.

9. Alali H, Braeckman L, Van Hecke T, et al. Relationship between nonstandard work arrangements and work-related accident absence in Belgium. J Occup Health 2017;59:177-86.

10. Dong X. Long workhours, work scheduling and work-related injuries among construction workers in the United States. Scand J Work Environ Health 2005;31:329-35.

11. Dong XS, Wang X, Largay JA. Occupational and non-occupational factors associated with work-related injuries among construction workers in the USA. Int J Occup Environ Health 2015;21:142-50.

12. Marucci-Wellman HR, Willetts JL, Lin TC, et al. Work in multiple jobs and the risk of injury in the US working population. Am J Public Health 2014;104:134-42.

13. Bena A, Berchialla P, Debernardi ML, et al. Impact of organization on occupational injury risk: evidence from high-speed railway construction. Am J Ind Med 2011;54:428-37.

14. Hintikka N. Accidents at work during temporary agency work in Finland - Comparisons between certain major industries and other industries. Saf Sci 2011;49:473-83.

15. Smith CK, Silverstein BA, Bonauto DK, et al. Temporary workers in Washington state. Am J Ind Med 2010;53:135-45.
16. Statistics Sweden (SCB). Labour force survey 2014. 2018. http:// www.scb.se/Statistik/AM/AM0401/2014K02/AM0401_2014K02_SM_ AM11SM1403.pdf

17. Cheng C-W, Leu S-S, Lin C-C, et al. Characteristic analysis of occupational accidents at small construction enterprises. Saf Sci 2010;48:698-707.

18. Boisjoly RP. The effects of financial performance and deregulation in the trucking and airline industries on worker Safety. New Solutions 1997;7:38-45.

19. Asfaw A, Mark C, Pana-Cryan R. Profitability and occupational injuries in U.S. underground coal mines. Accid Anal Prev 2013:50:778-86.

20. Benach J, Vives A, Amable M, et al. Precarious employment: understanding an emerging social determinant of health. Annu Rev Public Health 2014;35:229-53.

21. Benach J, Vives A, Tarafa G, et al. What should we know about precarious employment and health in 2025? Framing the agenda for the next decade of research. Int J Epidemiol 2016;45:232-8.

22. Swedish Work Environment Authority (Arbetsmiljöverket). Anmäl Arbetsskada. 2018. https://anmalarbetsskada.se/

23. Statistics Sweden (SCB). Swedish standard classification of occupations (SSYK96). Stockholm: Reports on Statistical Coordination for the Official Statistics of Sweden, 2018:1-191.

24. Statistics Sweden (SCB). Swedish Standard Industrial Classification (SNI). 2018. https://www.scb.se/dokumentation/klassifikationer-ochstandarder/standard-for-svensk-naringsgrensindelning-sni/

25. Hook EB, Regal RR. Capture-recapture methods in epidemiology: methods and limitations. Epidemiol Rev 1995;17:243-64.

26. Swedish Work Environment Authority (Arbetsmiljöverket), Arbetsskador. Occupational accidents and work-related diseases, in Report 2017. Stockholm: Swedish Work Environment Authority, 2016.

27. López-Ruiz M, Martínez JM, Pérez K, et al. Impact of road safety interventions on traffic-related occupational injuries in Spain, 20042010. Accid Anal Prev 2014;66:114-9.

28. Eurofund (European Foundation for the Improvement of Living and Working Conditions). European working conditions survey. 2015. https://www.eurofound.europa.eu/data/european-workingconditions-survey 